\title{
Long-Range Conformational Changes in Monoclonal Antibodies Revealed Using FPOP-LC-MS/MS
}

\author{
Owen Cornwell, ${ }^{\dagger}$ Nicholas J. Bond, ${ }^{\ddagger}$ Sheena E. Radford, ${ }^{\dagger}$ a and Alison E. Ashcroft* ${ }^{*}{ }^{\dagger}$ \\ ${ }^{\dagger}$ Astbury Centre for Structural Molecular Biology and School of Molecular and Cellular Biology, Faculty of Biological Sciences, \\ University of Leeds, Leeds, LS2 9JT, U.K. \\ ${ }^{\ddagger}$ Analytical Sciences, BioPharmaceutical Development, BioPharmaceuticals R\&D, AstraZeneca, Granta Park, Cambridge, CB21 \\ 6GH, U.K.
}

\section{Supporting Information}

ABSTRACT: Differences in conformational dynamics between two full-length monoclonal antibodies have been probed in detail using Fast Photochemical Oxidation of Proteins (FPOP) followed by proteolysis and LC-ESI-MS/ MS analyses. FPOP uses hydroxyl radical labeling to probe the surface-accessible regions of proteins and has the advantage that the resulting covalent modifications are irreversible, thus permitting optimal downstream analysis. Despite the two monoclonal antibodies (mAbs) differing by only three amino acids in the heavy chain complementarity determining regions (CDRs), one mAb, MEDI1912-WFL, has been shown to undergo reversible self-association at high concentrations and exhibited poor pharmacokinetic properties in vivo, properties which are markedly improved in the variant, MEDI1912-STT. Identifying the differences in oxidative labeling between the two antibodies at residue level revealed long-range effects which provide a key insight into their conformational differences. Specifically, the amino acid mutations in the CDR region of the heavy chain resulted in significantly different labeling patterns at the interfaces of the $C_{L}-C_{H} 1$ and $C_{H} 1-C_{H} 2$ domains, with the nonaggregating variant undergoing up to four times more labeling in this region than the aggregation prone variant, thus suggesting a change in the structure and orientation of the $\mathrm{C}_{\mathrm{L}}-\mathrm{C}_{\mathrm{H}} 1$ interface. The wealth of FPOP and LC-MS data obtained enabled the study of the LC elution properties of FPOP-oxidized peptides. Some oxidized amino acids, specifically histidine and lysine, were noted to have unique effects on the retention time of the peptide, offering the promise of using such an analysis as an aid to MS/MS in assigning oxidation sites.
$\mathrm{L}$ ong-range conformational interactions between the various domains of monoclonal antibodies (mAbs), particularly the variable (V) and constant (C) domains, has been a hotly debated topic since the 1970s. ${ }^{1-3}$ Although numerous studies have suggested a functional allosteric link between antigen binding in the $\mathrm{V}$ domains of the antigenbinding fragment $(\mathrm{Fab})$ and $\mathrm{C}$ domain function in the fragment crystallizable $(\mathrm{Fc})$ region (reviewed in ref 2), the prevailing hypothesis is that the $\mathrm{Fab}$ and $\mathrm{Fc}$ domains are functionally independent, ${ }^{4,5}$ supported by the perceived difficulty of transmitting allosteric conformational changes via the highly flexible hinge connecting the two regions. ${ }^{6}$

However, recent mounting evidence suggests that long-range conformational changes, either as a result of antigen binding or amino acid substitutions, can be transmitted between $\mathrm{C}$ and $\mathrm{V}$ domains within the Fab region of an antibody. It is now wellestablished that class switches in the $\mathrm{C}$ domain of Fab arms can affect antigen binding affinity ${ }^{7-10}$ and instigate changes in cooperative unfolding. ${ }^{11}$ Further, class switches in either the $\mathrm{C}$ or $\mathrm{V}$ domains can change the elbow angle of the Fab arm. ${ }^{7,12}$ Together these effects suggest that the conformation of the antigen binding site can, to some extent, be modulated by changes in the $\mathrm{C}$ domain, via long-range conformational changes mediated via the elbow angle.' Other studies suggested that the reverse communication is also possible, whereby antigen binding may change the conformation of the $\mathrm{C}$ domains. ${ }^{13}$ Indeed, a systematic comparison of antigenbound and antigen-free $\mathrm{mAb}$ structures in the Protein Data Bank (pdb) revealed conformational changes in the relative orientation of $\mathrm{V}$ and $\mathrm{C}$ domains in the Fab for both heavy $(\mathrm{H})$ and light (L) chains, as well as significant movement of the $\mathrm{C}_{\mathrm{H}} 1-1$ loop, an essential component of the $\mathrm{C}_{\mathrm{H}} 1-\mathrm{C}_{\mathrm{L}}$ interface, ${ }^{14}$ suggesting that long-range conformational changes within the Fab arm can be instigated from either direction.

Surprisingly, subtle changes, such as single amino acid substitutions, have also been observed to cause long-range conformational changes in Fabs. Individual point mutations at the $C_{L}-V_{L}$ interface have been shown to change thermal

Received: August 29, 2019

Accepted: November 5, 2019

Published: November 5, 2019 
stability and antigen binding, as well as the hydrogendeuterium exchange (HDX) behavior, of the complementarydetermining regions (CDRs). ${ }^{15}$ More recently, Henderson et al. demonstrated that a double mutation of amino acid sidechains in the elbow and framework regions of the $\mathrm{V}$ domain resulted in major increases in flexibility of the elbow joint and in the more distant CDR loops of both the $\mathrm{H}$ and $\mathrm{L}$ chains. ${ }^{16}$ Robust analytical methods to characterize such long-range conformational effects are crucial for our further understanding of antibody function, and for the rational design of effective biotherapeutics.

Fast photochemical oxidation of proteins (FPOP) is emerging in the field of structural MS as a valuable method with which to probe solvent accessibility and protein structure. $^{17-21}$ FPOP employs hydroxyl radicals, liberated from photolysis of hydrogen peroxide, to covalently label solvent-accessible amino acid side-chains (preferentially labeling aromatic or sulfur containing groups ${ }^{22}$ ), on the $\mu \mathrm{s}$-ms time scale. $^{23-25}$ Following proteolysis and LC-MS/MS, the resulting covalent oxidative modifications, the most common of which are $+16 \mathrm{Da},+32 \mathrm{Da}$, and $+14 \mathrm{Da}$, can be localized to residuelevel resolution using standard ergodic fragmentation techniques, such as collision induced dissociation (CID). ${ }^{17}$ As such, FPOP offers many advantages over alternative structural MS techniques, such as hydrogen-deuterium exchange (HDX), where single residue resolution is typically difficult to achieve, ${ }^{26}$ and conditions required to minimize back exchange of the reversible deuterium label limit the available analysis time and choice of proteases for digestion. ${ }^{17}$

However, downstream analysis and interpretation of FPOPLC-MS/MS experimental data is highly complex. For example, while the effects of sequence and nearby side-chains on the degree of labeling are well-established in HDX-MS, ${ }^{27,28}$ these aspects are currently less well-defined for FPOP experiments. ${ }^{17,21}$ Similarly, FPOP analysis strategies to identify modified residues currently rely solely on tandem MS, which can be challenging given the inherent low abundance of most oxidations and the multitude of isobaric products. ${ }^{29,30}$ Despite this, FPOP has not only shown great promise with regards to structural characterization of mAbs in epitope mapping, ${ }^{18,20,30-32}$ but has also been used to detect minor conformational changes, distant to binding sites in both the $\mathrm{Fc}^{33}$ and CDR regions, ${ }^{18}$ and recently has been used to identify changes in solvent-accessible surface area of amyloid proteins at subamino acid level resolution. ${ }^{17}$

Here, we explore the utility of FPOP to characterize MEDI1912, a human IgG1 mAb selected against nerve growth factor, and its variant containing three amino acid substitutions in the heavy chain CDRs: W30S, F31T, and L57T. Hereafter the $\mathrm{mAbs}$ are referred to by their amino acid substitutions: WFL (original variant) and STT (triple mutation variant). ${ }^{34}$ Although the introduction of the amino acid substitutions resulted in no significant loss in antigen affinity (both have $\mathrm{pM}$ $K_{\mathrm{d}}$ ), STT showed a significantly reduced propensity to undergo reversible self-association, had a 3-fold slower clearance rate in vivo, and a reduced tendency for nonspecific tissue association. ${ }^{34}$ The two variants respond differently under stresses from shear and extensional flow ${ }^{35}$ and, although HDX and cross-linking data suggested a possible interface for the reversible self-association of WFL, ${ }^{34}$ the intramolecular conformational changes associated with this triple mutation have yet to be explored.
Using the extensive data sets generated by studying such large proteins, we aimed to gain a better understanding of the sensitivity and applicability of FPOP to probe long-range conformational changes in mAbs, to appreciate the extent to which our current understanding of the technique allows us to interpret structural changes, and to determine the extent to which trends in LC retention time can be used to aid MS/MS in assigning oxidations at the residue level.

\section{EXPERIMENTAL SECTION}

Fast Photochemical Oxidation of Proteins (FPOP). The FPOP experimental setup used was as described previously. ${ }^{19}$ The mAb proteins, WFL and STT, were supplied by AstraZeneca (Cambridge, UK) in formulation buffer (125 $\mathrm{mM}$ L-arginine in $20 \mathrm{mM}$ sodium succinate buffer, $\mathrm{pH} 6.0$ ). Due to the radical scavenging effect of the arginine excipient, ${ }^{22}$ oxidation conditions were reoptimized from conditions previously used in our group. ${ }^{17}$ Immediately prior to irradiation, $3 \mu \mathrm{L}$ of $5 \% \mathrm{v} / \mathrm{v} \mathrm{H}_{2} \mathrm{O}_{2}$ was added to $100 \mu \mathrm{L}$ of solution containing the analyte protein at $0.1 \mathrm{mg} \mathrm{mL} \mathrm{mL}^{-1}$, concentrations at which both proteins have been shown to be predominantly monomeric, ${ }^{34}$ in the formulation buffer containing an additional $10 \mathrm{mM}$ L-histidine scavenger. Following irradiation, the sample was collected in an Eppendorf vial containing $20 \mu \mathrm{L}$ quench solution $(100 \mathrm{mM}$ L-methionine, $1 \mu \mathrm{M}$ catalase in the formulation buffer) and placed immediately on ice. Control experiments under identical conditions with the exception that the proteins were not subjected to laser irradiation were carried out.

Protein Digestion. Disulfide bonds were reduced by incubation with dithiothreitol $\left(1 \mathrm{~h}, 65^{\circ} \mathrm{C}, 500 \mathrm{rpm}\right)$, before alkylation with iodoacetamide (incubated in the dark for 45 min, $\left.20{ }^{\circ} \mathrm{C}, 500 \mathrm{rpm}\right)$ and trypsin digestion $(1: 50 \mathrm{w} / \mathrm{w}$ enzyme/protein, incubated at $37{ }^{\circ} \mathrm{C}$, for $18 \mathrm{~h}$ at $\left.500 \mathrm{rpm}\right)$.

ESI-MS Analyses. To verify that the intact proteins had undergone photolytic oxidation, molecular masses were determined using ESI-MS on a Xevo mass spectrometer (Waters Corpn., Manchester, UK).

LC-MS/MS Analyses. Tryptic peptides $(1 \mu \mathrm{L}$ at $0.5 \mu \mathrm{M}$ peptide concentration) were injected onto a UPLC M-Class nano-Acquity system equipped with a $\mathrm{C} 18$ column $(75 \mu \mathrm{m} \times$ $150 \mathrm{~mm}$, Waters Corpn., Manchester, UK) and separated using reverse-phase chromatography with a linear gradient of $1-60 \%$ $\mathrm{v} / \mathrm{v} \mathrm{MeCN}$ in $\mathrm{H}_{2} \mathrm{O}$ (both containing $0.1 \% \mathrm{v} / \mathrm{v}$ formic acid) over $90 \mathrm{~min}$ at $0.3 \mu \mathrm{L} \mathrm{min}{ }^{-1}$. Peptides were analyzed using ESI-MS/MS on an Orbitrap Fusion mass spectrometer (ThermoFisher Scientific, Bremen, Germany) operating in Orbitrap-iontrap mode (240000 resolution, rapid) with the following data dependent acquisition settings: $\operatorname{Top} N=7$, maximum injection time $=200 \mathrm{~ms}$, apex detection $=$ on. Dynamic exclusion was reduced to $3 \mathrm{~s}$ to allow MS/MS fragmentation of isobaric FPOP modifications with similar retention times.

Peptide Identification. Peptides were identified using PEAKS X software (Bioinformatics Solutions Inc., Waterloo, ON, Canada). Peptides were searched with a $10 \mathrm{ppm}$ mass error cut off and a false discovery rate of $0.1 \%$. The following oxidized mass additions were searched: $+14 \mathrm{Da}\left(\mathrm{CH}_{2}\right.$ to $\left.\mathrm{CO}\right)$, +16 $\mathrm{Da}(\mathrm{O}),+32 \mathrm{Da}(2 \mathrm{O}),+73 \mathrm{Da}$, and +89 Da (the latter two being singly and doubly oxidized Cys following carbamidomethylation). Quantification of modified peaks was performed manually using Xcalibur software (ThermoFisher Scientific, Bremen, Germany) by integration of peaks 
generated from extracted ion chromatograms (XICs) using the following equation:

$$
\% \text { modified }=\frac{\sum \text { modified }_{\text {peak }}}{\text { unmodifed }+\sum \text { modified }_{\text {allpeaks }}}
$$

All observed charge states were quantified for each peptide. XICs were generated by extracting over the base peak of each peptide isotope distribution, for each charge state. Unless stated otherwise, residue level quantification data are combined from all observed positional isomers for a single amino acid residue. Where MS/MS was insufficient to localize the modification to individual side-chains, the sequence of amino acids to which the modification could be localized, are shown. Modified peaks with retention times indistinguishable from that of the unmodified peptide were excluded from quantification as the possibility that these resulted from insource oxidations, rather than genuine FPOP modifications, could not be ruled out. $t$ tests were used to determine statistically significant changes, where $p<0.01$ was determined to be statistically significant.

Structural Modeling of mAbs. Homology models of both the $\mathrm{Fab}$ and $\mathrm{Fc}$ domains of full length WFL were generated using SWISS-MODEL. ${ }^{36}$ The Fab homology model was generated using pdb: $5 \mathrm{wkz}^{37}$ (sequence identity = 95.23\%). The Fc homology model was generated using pdb: $5 \mathrm{k}^{2} 5^{38}$ (sequence identity $=89.81 \%$ ). These were then aligned into the canonical ' $\mathrm{Y}$ ' shape of an IgG using pdb: $1 \mathrm{IGY}{ }^{39}$

\section{RESULTS AND DISCUSSION}

Comparison of mAbs WFL and STT using FPOP LCMS/MS. The two mAbs, WFL and STT, were subjected individually to photochemical oxidation using FPOP. In each case, control experiments in which the protein was treated with hydrogen peroxide but not exposed to laser irradiation were carried out to ensure that only FPOP oxidation was assessed. Following tryptic digestion of the oxidized and control proteins, LC-ESI-MS/MS analysis of the resulting peptides yielded $80 \%$ and $96 \%$ sequence coverage for WFL, and $82 \%$ and $100 \%$ sequence coverage for STT, for the heavy and light chains, respectively (Supporting Information (SI) Figures S1 and S2). Across both the heavy and light chains, 50 different oxidized sites were identified ( 28 on the heavy chain, 22 on the light chain), 45 of which were common to both WFL and STT.

The most obvious differences in identified modification sites were directly proximal to the W30S and F31T mutations in the heavy chain CDRs of the mAbs. The extracted ion chromatograms (XICs) for the modified and unmodified versions of the WFL peptide covering residues $24-38$ in this region (Figure 1a,b) show multiple modified species for both W30 and F31 eluting at different retention times. These isobaric oxidized species, corresponding to the $+16 \mathrm{Da}$ incorporation of a single oxygen atom, indicate multiple positional isomers resulting from hydroxyl radical incorporation at different positions on the aromatic rings of the tryptophan and phenylalanine sidechains. ${ }^{17}$ Additionally, in some instances, W30 was found to undergo two oxidations within the same side-chain resulting in the overall addition of $32 \mathrm{Da}$.

Despite a prominent y ion series, commonly seen for tryptic peptides $^{40}$ (typically basic at their C terminus), for both WFL and STT, several oxidized products were observed to have ambiguous MS/MS data, localizing the modification to between residues 35 and $37(35-\mathrm{TWV}-37)$ or to residues
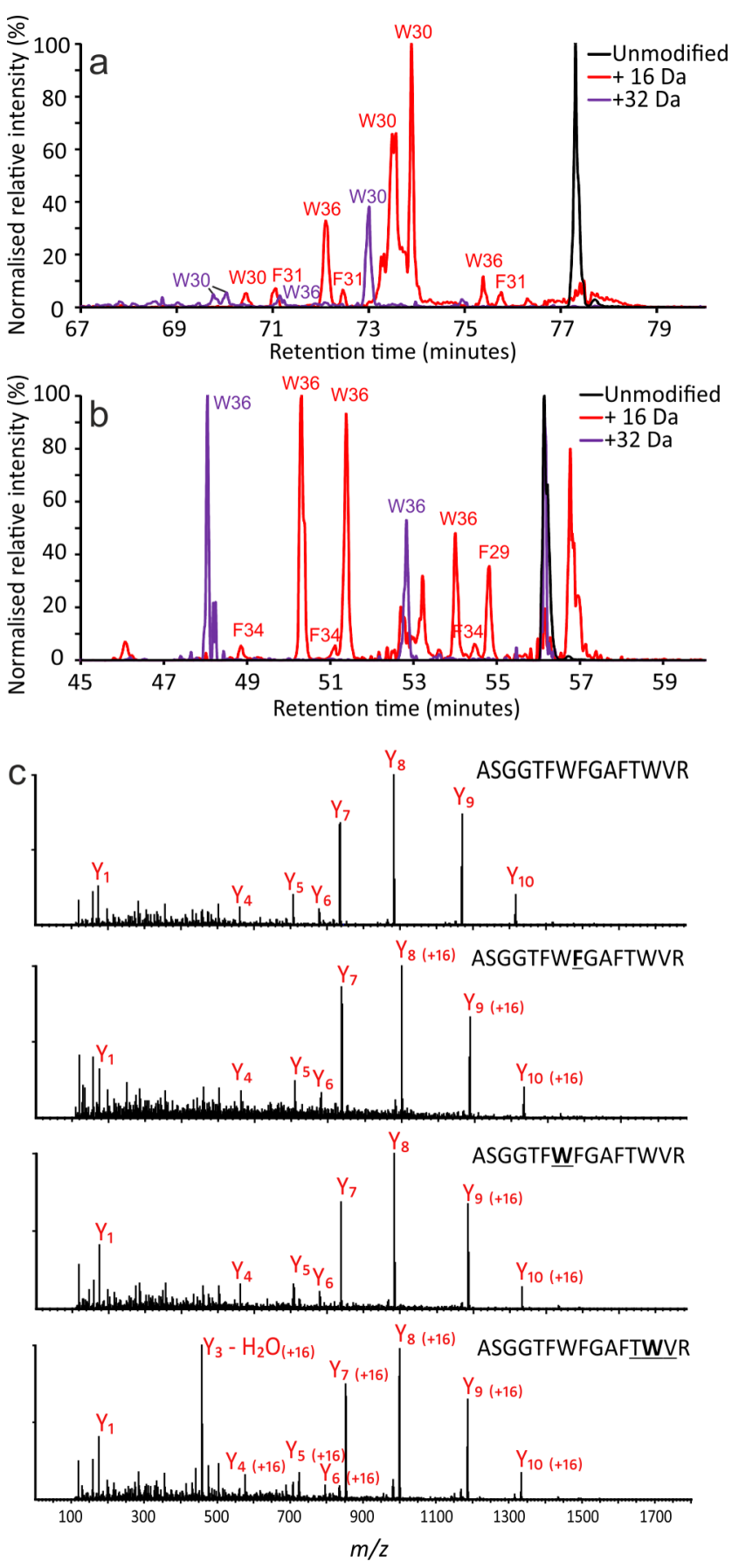

Figure 1. FPOP oxidations in the region surrounding the W30S and F31T mutation sites. XICs for the unmodified (black), +16 Da modified (red) and +32 Da doubly modified (purple) versions of each peptide for (a) WFL (24-ASGGTFWFGAFTWVR-38) and (b) STT (24-ASGGTFSTGAFTWVR-38). Peaks are annotated according to which oxidized residue was identified using MS/MS. (c) Representative tandem MS spectra for unmodified (top) and modified (bottom three) WFL peptides covering the region of WF substitutions. The residue(s) in which the modification site was identified using MS/MS are underlined, with the assigned modified residue highlighted in bold on the sequence on each spectrum.

W36 or V37 if water loss fragment ions are used for assignment ${ }^{41}$ (Figure 1c). Owing to the significant reactivity bias of side-chains in this remnant sequence, in addition to the presence of multiple isobaric products, as well as double oxidations, these modifications most likely arise from the 

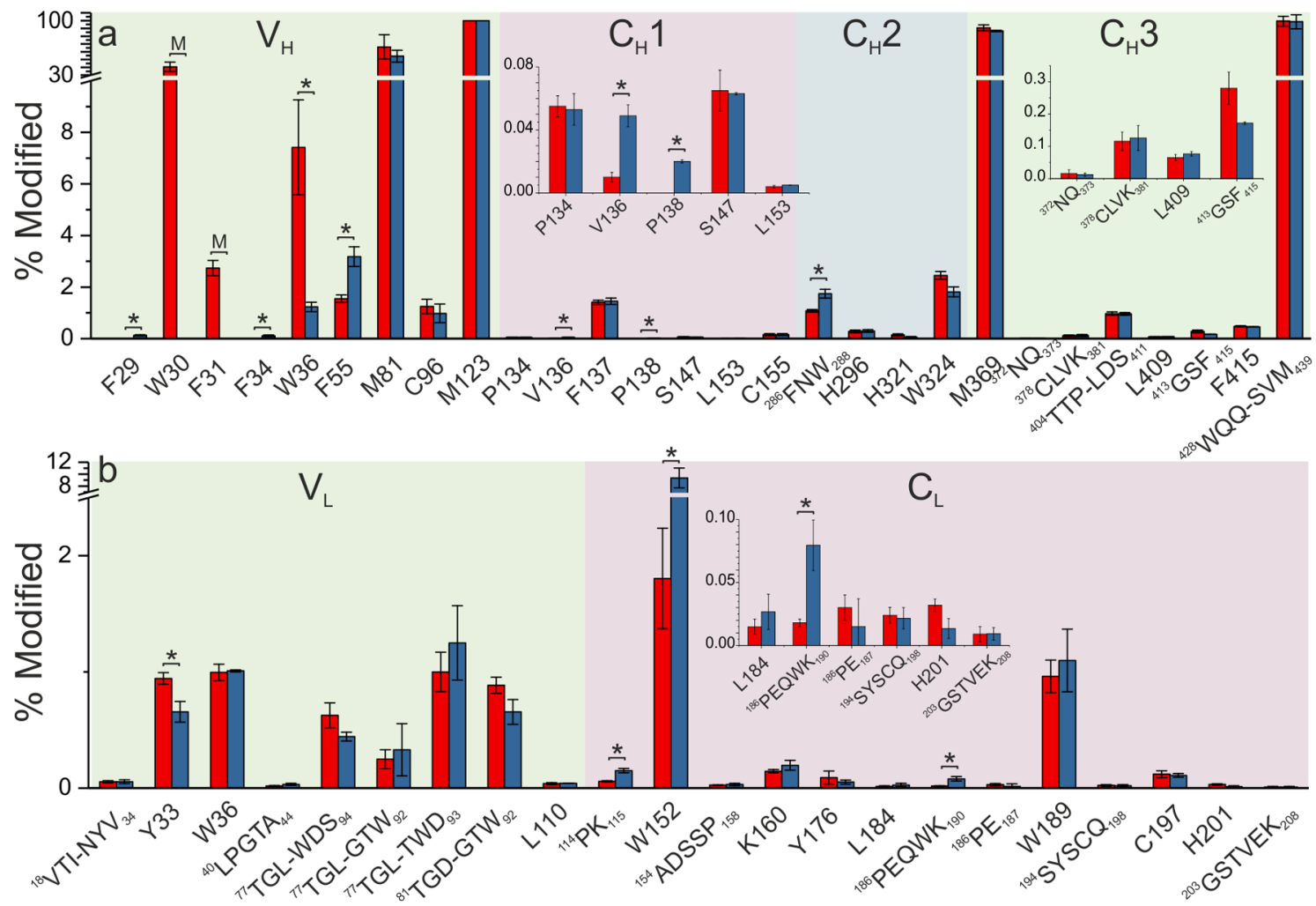

Figure 2. Quantification of oxidized sites of the mAbs WFL and STT. Sites of modification for WFL (red) and STT (blue) for (a) the heavy and (b) the light chains are shown along the $x$ axis. Modifications are localized to a residue, or short sequence of amino acids. Where the modification could not be localized to a sequence of $<6$ amino acids, the first and last three residues in the sequence are highlighted. Mutation sites which were found to be oxidized in either variant (W30S and F31T) are annotated with "M". Inserts show low abundance modifications on rescaled axes. * $=p$ $<0.01$; error bars show standard deviation $(N=3)$.

oxidation of $\mathrm{W} 36 .^{22}$ Surprisingly, despite being a core residue of the $V_{\mathrm{H}}$ domain, this side-chain was $\sim 6$-fold more labeled in WFL than STT (Figure 2a), which suggests a notable change in conformation and solvent accessibility of side-chains in this region. Indeed, further examination of oxidized products in this region lends weight to this hypothesis. F29 and F34, residues either side of the mutation sites, are only observed to be modified in the STT variant (Figure 1b). Similarly, F55, present in the nearby $V_{\mathrm{H}} \mathrm{CDR} 2$ region and adjacent to the mutation site L57, was observed to increase in labeling by $\sim 2$ fold in the STT variant (Figure 2a). Together, these findings may suggest a structural rearrangement of the $V_{H}$ domain, proximal to the heavy chain CDRs, in which all four of these side-chains have altered solvent accessibility as a result of the WFL-STT substitutions. However, recent evidence suggests that side-chain microenvironment and amino acid sequence can play a significant role in determining the degree to which side-chains are susceptible to radical oxidation. ${ }^{17,21,42}$ The W30S, F31T, and L57T substitutions are expected to decrease the reactivity to hydroxyl radical labeling of these individual amino acid positions by factors of 40,13 , and 3, respectively. ${ }^{22}$ This likely explains the absence of modification for two of the equivalent amino acid positions in STT (S30 and T31; residue 57 labels in neither variant). Importantly, the substitution of the FPOP reactive $\mathrm{W} 30$ and F31 side-chains for less oxidationprone residues may also explain the increased oxidation of the nearby F55 and W36 side-chains in the STT variant, as well as the oxidation of F29 and F34 seen solely in the STT variant, which could be as a result of diminished competition for hydroxyl radicals in the region. F29, F35, and F55 are all within $\sim 10 \AA$ of the mutation site, hence hydroxyl radical attack on nearby, less reactive/less solvent exposed groups in the region could be expected to be more likely in STT. Recent reports have suggested that hydrogen peroxide preferentially interacts with certain side-chains, including threonine, but not leucine residues. $^{43,44}$ As such, an alternative explanation for the increased modification on F55 in STT, is that the L57T mutation increases the local concentration of hydrogen peroxide. Although the effects of local microenvironment in FPOP have not been fully explored, the local microenvironment changes discussed here provide an alternative explanation as the cause of the changes in oxidation observed in this region.

Four other modification sites, each with a $p$ value $<0.01$, showed a $>4$-fold difference in labeling between the two $\mathrm{mAb}$ variants, all of which indicate significantly lower labeling in WFL. These residues are P138 (unlabeled in WFL) and V136 in the $\mathrm{C}_{\mathrm{H}} 1$ domain (Figure 2a), in addition to W152 and an unidentified modification between P186 and K190 (186PEQWK-190) in the $\mathrm{C}_{\mathrm{L}}$ domain (Figure 2b). Interestingly, all four of these sites are found to occupy a region in the lower half of the $C_{L}$ domain, at the $C_{L}-C_{H} 1$ interface (Figure 3 ). Minor oxidation changes between WFL and STT $(<2$-fold) could also be localized to 286-FNW-288 (peptide 286FNWYVDGVEVHNAK-299) at the top of the nearby $\mathrm{C}_{\mathrm{H}} 2$ domain, again showing lower labeling in the WFL variant (Figure 3). These oxidation differences between the WFL and STT variants were surprising, as they are $>60 \AA$ from the introduced mutations in the CDRs of the heavy chains of these mAbs, and suggest either a conformational change of the 


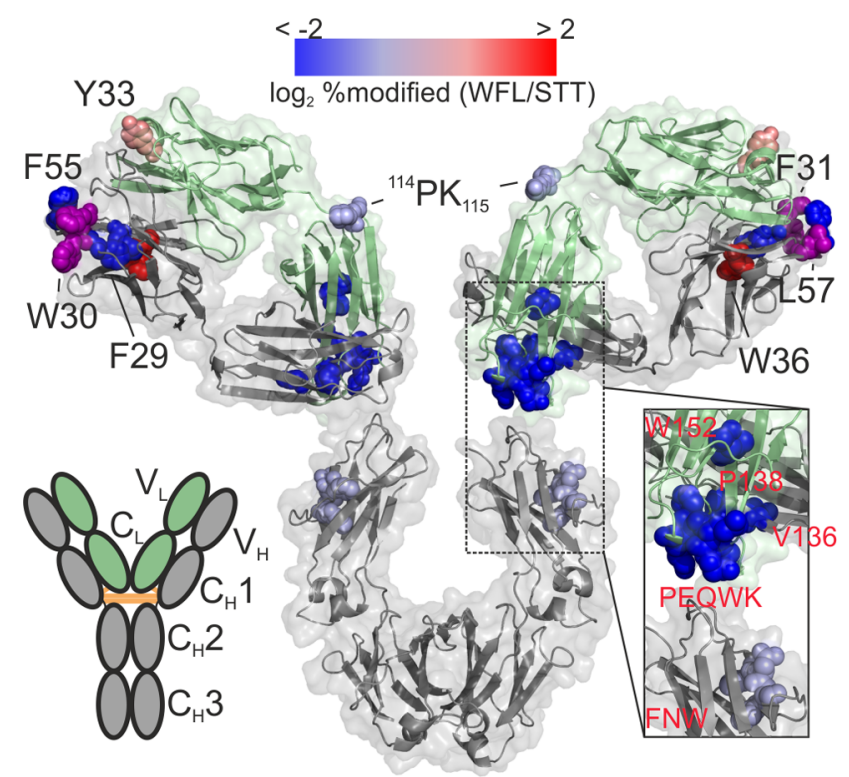

Figure 3. Differences between WFL and STT following FPOP oxidation. Significantly different modified sites $(p<0.01)$ are indicated by spheres and colored by the magnitude of the difference observed. The heavy and light chains are depicted by gray and green ribbons, respectively. The mutations (W30S, F31T, L57T) are shown as purple spheres. Insert: zoomed image of the $\mathrm{C}_{\mathrm{L}}-\mathrm{C}_{\mathrm{H}} 1$, and $\mathrm{C}_{\mathrm{H}} 1-$ $\mathrm{C}_{\mathrm{H}} 2$ interfaces, where significant conformational changes were found between WFL and STT. Oxidation sites are annotated in red letters either to the residue level, or to a short sequence to which MS/MS could localize the modification. Details of the WFL homology model generated here can be found in SI Figure S3.

constant domains, or a change in orientation of the $C_{L}-C_{H} 1$ domains relative to one another.

Changes in the $C_{L}-C_{H} 1$ interface thought to be related to antigen binding have been reported elsewhere, ${ }^{14}$ suggesting a conformational link between the two regions. One report demonstrated that a class switch of the $\mathrm{C}_{\mathrm{L}}$ domain caused a change in orientation of the $C_{L}-C_{H} 1$ domains associated with changes in CDR loop structure, which altered the surface exposed hydrophobicity of that region. ${ }^{7}$ The data presented here suggest a similar trend in reverse, whereby amino acid substitutions that alter the surface-exposed hydrophobicity of the CDRs elicit changes in $\mathrm{C}_{\mathrm{L}}-\mathrm{C}_{\mathrm{H}} 1$ orientation. It has been suggested that conformational changes between the $\mathrm{C}$ and $\mathrm{V}$ domains of Fab arms occurs through the elbow angle between the two domains. ${ }^{9}$ Although no modifications were identified in the elbow region between the $\mathrm{V}_{\mathrm{H}}$ and $\mathrm{C}_{\mathrm{H}} 1$ domains in our study, one modified species was identified in the elbow region between the $\mathrm{V}_{\mathrm{L}}$ and $\mathrm{C}_{\mathrm{L}}$ domains, localized to P114/K115, where $\sim 2.5$-fold lower labeling was observed in WFL compared with STT $(p<0.01$, Figure $2 \mathrm{~b})$.

Perhaps the most striking difference in oxidative labeling was observed for W152 in the light chain, located on the edge of the $\mathrm{C}_{\mathrm{L}}$ domain (Figure 3). At the residue level, W152 showed $\sim 5$-fold less oxidation for WFL than for STT (Figure 2b). However, closer examination of the XIC revealed five distinct peaks at different retention times, all corresponding to modified W152, resulting from the incorporation of oxygen at different positions on the side-chain, generating isobaric positional isomers ${ }^{17,22}$ (Figure 4a). Previous studies have shown that, in simpler systems, detailed examination and quantification of individual tryptophan positional isomers in
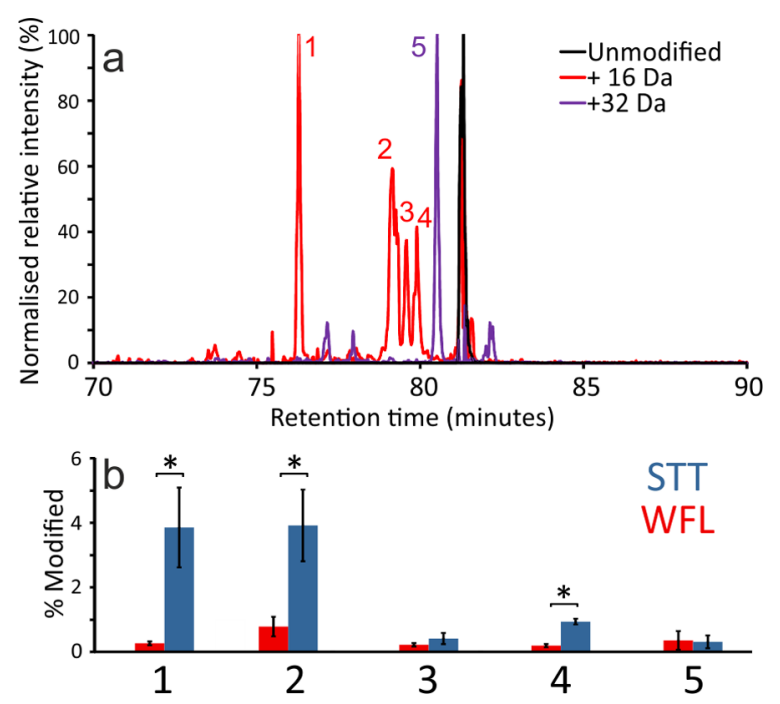

Figure 4. FPOP quantification of the light chain W152 positional isomers. (a) XICs of the unmodified (black), $+16 \mathrm{Da}$ (red) and +32 $\mathrm{Da}$ (purple) modified peptide 134-153. Isobaric peaks $1-4$ had a single (+16 Da) oxidation; peak 5 had two oxidations $(+32 \mathrm{Da})$. (b) Quantification of the modified species, with the same peak 1-5 annotation as (a), for WFL (red) and STT (blue). Significantly different modified sites $(p<0.01)$ are indicated with an *. Error bars show standard deviation $(N=3)$.

FPOP can yield higher resolution structural information, effectively offering subamino acid level resolution on changes in protein structure for certain side-chains. ${ }^{17}$ Although no robust method of fully characterizing positional isomers following FPOP yet exists, and more work is needed to establish the utility of these products in determining changes in protein structure, separate quantification of the W152 isomers observed here can provide some additional information on the conformational changes for this side-chain.

Three of the four observed $+16 \mathrm{Da}$ isomers of W152 show significantly lower labeling in WFL than in STT by up to $\sim 14 \times$, indicative of a drastic change in the environment of this side-chain between the two variants (Figure 4b). However, the remaining +16 Da isomer, as well as the doubly oxidized +32 Da product, exhibit little change (Figure $4 \mathrm{~b}$ ). Given that the relationship between changes in solvent accessibility and the associated changes in modification for different side-chains has yet to be established in FPOP, and is likely complicated by the effects of the microenvironment discussed earlier, determining the precise magnitude of the change in solvent accessibility for W152 is challenging. However, the observation that some of the W152 oxidation products are similar between the two variants, could suggest that part of the W152 side-chain remains buried in both WFL and STT. Relatedly, several oxidized residues similarly proximal to the $\mathrm{C}_{\mathrm{L}}-\mathrm{C}_{\mathrm{H}} 1$ interface also show similar degrees of labeling between the two variants. For example, F137 in the heavy chain, and W189 and K160 in the light chain all show no statistically significant change, Figure $2 a$ and $b$ ). Together, these data could indicate a more subtle rearrangement of the $\mathrm{C}_{\mathrm{L}}-\mathrm{C}_{\mathrm{H}} 1$ interface, and highlight the sensitivity of FPOP to identify minor conformational changes and side-chain movements, which typically are difficult to probe by lower resolution structural MS techniques, such as HDX.

Insights from FPOP LC-MS/MS Retention Time Data. Proteolytic digests, and subsequent LC-MS/MS data, from 
proteins oxidized by FPOP are complex, where multiple different oxidized products, eluting at different retention times, can be observed for each peptide, corresponding to different oxidized residues within the peptide, as well as different positional isomers of the same oxidized residue. Currently, identification of the sites of FPOP oxidation is achieved by interpretation of the MS/MS data, where LC separation prior to MS is used primarily to separate peptides over time, allowing more fragmentation spectra to be acquired, and minimizing the occurrence of difficult to interpret, chimeric tandem MS spectra, resulting from overlapping, or partially separated, isobaric modified peptides. Although retention time change for oxidized peptides is a well observed phenomenon, ${ }^{17}$ and optimal peptide separation prior to MS is, at present, an essential requirement for adequate assignment and quantification of oxidations in FPOP experiments, the LC separation data has, to date, not been systematically investigated. Here, we sought to explore the possibility that the LC separation data, in conjunction with MS/MS, could be used as an aid in identifying modified residues via trends in changes in retention time for certain modified side-chains.

Retention time data were compiled from the most common oxidative modifications identified unambiguously to the residue level using MS/MS both from previous work in our laboratory, ${ }^{17}$ as well as the data acquired in this study. This allowed us to analyze the effects of different oxidative modifications on the retention time of the peptide. Using reverse-phase $\mathrm{LC}$ with an aqueous $\mathrm{MeCN}$ gradient, most peptides with oxidative modifications were observed to elute at lower $\mathrm{MeCN}$ concentrations (shorter retention times) compared with their unmodified counterparts, as expected, given the incorporoation of an oxygen atom and subsequent decrease in hydrophobicity of the peptide. However, modified peptides on which the oxidation was present on a histidine side-chain were observed to elute reproducibly at higher $\mathrm{MeCN}$ concentrations (later retention times) than their unmodified peptide counterparts (Figure 5a), an observation made previously by us and others ${ }^{19,32}$ but without further details.

Histidine oxidation has previously been reported to render protonation of the side-chain significantly less favorable, likely due to the considerably lower proton affinity of the dominant +16 Da oxidation product, 2-oxo-histidine (Figure 5b). ${ }^{22,45-47}$ This suggests the 2-oxo-histidine product is likely to remain unprotonated under typical acidic LC conditions, likely resulting in increased hydrophobicity of the modified peptide compared to the unmodified version, despite the incorporation of an oxygen atom and formation of a carbonyl group. This hypothesis is supported by the observation that histidine modifications, unlike modifications of other side-chains, can show significant charge state bias toward lower charge states (Figure 5b), although this bias was not observed in all cases where histidine was the modified residue, and likely depends on the presence of other protonatable groups on the peptide. Similarly, we have found that oxidative modifications on lysine residues have a reproducible effect on retention time, resulting in peptide elution immediately prior to the unmodified species, typically at an $\mathrm{MeCN}$ concentration $0.05-0.1 \%$ lower than the unmodified peptide (Figure 5a). This is consistent with a minimal effect of increased hydrophilicity on an already hydrophilic side-chain. These observations highlight the importance of quantifying all observable charge states when analyzing FPOP data, and that the characteristics of oxidatively
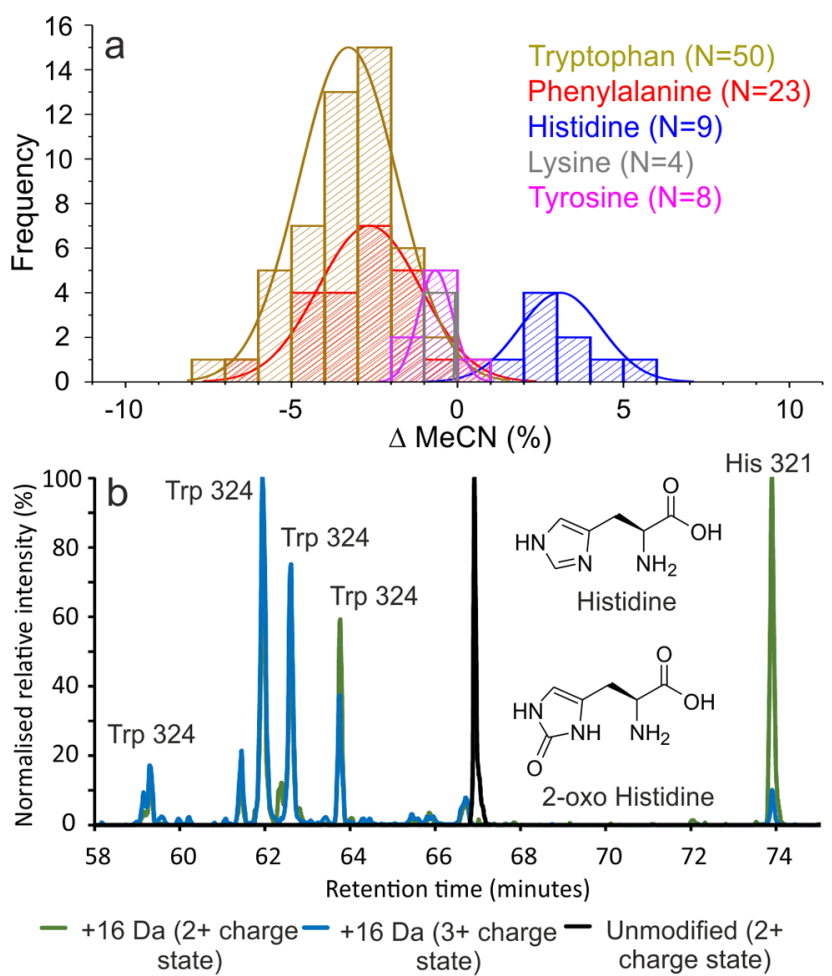

Figure 5. Retention time analysis of FPOP oxidized peptides. (a) Combined data analysis using retention time data from this study and previous work in our laboratory ${ }^{17}$ identifies trends in the change in retention time of the peptide in the presence of specific modifications. The retention time of the unmodified peptide is set at 0 . Histograms, binned every $\Delta 1 \% \mathrm{MeCN}$, are shown as shaded columns. Gaussian fits of the raw data are shown as solid lines. Data used for this analysis only include modifications that could be assigned unambiguously to the residue level by MS/MS. (b) XICs for the unmodified (black), and the $2+$ and $3+$ charge states (green and blue, respectively) of the +16 Da modified peptide 313-328 from the heavy chain of the mAbs. Annotations above each peak show which side-chain was modified, as determined by MS/MS.

labeled peptides can be used, along with MS/MS data, to support the identification of modified residues.

We have also noted a number of instances in which some oxidized peptides, with a range of modified residues identified from tandem MS spectra, eluted with precisely the same retention time as the unmodified peptide (SI Figure S4). One feasible explanation for this is that low intensity modifications of oxidation-prone side-chains may occur during the ESI process, that is, after LC separation, ${ }^{48,49}$ accounting for the identical retention times of a peptide in its unmodified and modified forms. Although the prospect of oxidized and unmodified versions of the same peptide eluting at the same retention time cannot be ruled out, the possibility of these "insource" oxidations means that care should be taken when interpreting such results, and further illustrates the importance of utilizing the LC separation data when assigning FPOP oxidations.

\section{CONCLUSIONS}

The results presented support the view that long-range conformational changes can occur between the $\mathrm{C}$ and $\mathrm{V}$ domains of the Fab region of antibodies, as evidenced by the conformational changes observed, in our study, at the $\mathrm{C}_{\mathrm{L}}-\mathrm{C}_{\mathrm{H}} 1$ interface, caused by the triple mutation in the heavy chain 
CDRs. Previous studies using FPOP and other hydroxyl radical foot printing methods have observed long-range changes in oxidative labeling as a result of binding to both the CDRs ${ }^{18}$ and the $\mathrm{Fc}_{\mathrm{c}}$ regions. ${ }^{33}$ These data, and those presented here, highlight the sensitivity of FPOP to identify minor conformational or side-chain solvent accessibility changes that could be vital in protein allostery and protein-protein interactions. However, the magnitude of the conformational rearrangement observed at the $\mathrm{C}_{\mathrm{L}}-\mathrm{C}_{\mathrm{H}} 1$ interface here cannot reliably be determined from these data. While several side-chains in the region show $>4$ fold difference in labeling, other nearby groups show no change between the two variants. Interpretation of FPOP data in this regard would benefit greatly from increased understanding of the relationship between solvent accessible surface area and the degree of modification observed, as well as the effects of microenvironment ${ }^{17,21}$ and the effect these factors have on the observed positional isomers. ${ }^{17}$ Similarly, the significance of these conformational differences with regards to the poor pharmacological properties of WFL cannot be established from these data. However, it has been noted recently that for light chain dimers, structurally similar to the $\mathrm{Fab}$ domains in antibodies, ${ }^{50}$ the $\mathrm{C}_{\mathrm{L}}$ domains modulate aggregation of the full length protein, whereas the interface between the constant domains was suggested as a potential target for preventing aggregation in light chain amyloidosis. ${ }^{51}$ Therefore, it seems plausible that the conformational differences we observe between the WFL and STT mAbs at the interface between the $\mathrm{C}_{\mathrm{L}}-\mathrm{C}_{\mathrm{H}} 1$ domains of the $\mathrm{Fab}$ may contribute to the propensity of WFL to form aggregates, and undergo reversible self-association. ${ }^{34,35}$

The retention time analysis of oxidized peptides in this study also reveals the potential for using the LC separation as a supportive analysis tool in identifying oxidized residues. Although fragmentation spectra are undoubtedly the gold standard in identifying FPOP oxidations, retention time prediction of peptides in reverse-phase LC has long been established, ${ }^{52}$ and the data presented here demonstrate the possibility of establishing a similar analysis to aid in identifying oxidation sites in FPOP. Further, positional isomers of oxidized aromatic residues have been shown to elute with a predictable retention time order using reverse-phase chromatographic separation, both in short peptides and as free amino acids. ${ }^{53,54}$ As such, retention time analysis and prediction could be a useful future tool for characterizing positional isomers in FPOP experiments.

The utility of FPOP as a method of probing protein structure and dynamics has now been demonstrated with regards to epitope mapping in biotherapeutics ${ }^{18,20,30-32}$ and the structural characterization of other systems aggregation prone protein proteins ${ }^{17}$ and studies into protein folding. ${ }^{19,55}$ Although more work is needed to advance FPOP further, both in method development, and in data analysis and interpretation, ${ }^{56}$ the sensitivity to conformational changes demonstrated here illustrate that FPOP can be a powerful analytical probe in the structural MS toolbox.

\section{ASSOCIATED CONTENT}

\section{S Supporting Information}

The Supporting Information is available free of charge on the ACS Publications website at DOI: 10.1021/acs.analchem.9b03958.
Sequence coverage maps for proteolytic digests of WFL and STT, homology model data, and LC and MS/MS data for suspected in-source oxidation (PDF)

\section{AUTHOR INFORMATION}

\section{Corresponding Author}

*E-mail: A.E.Ashcroft@leeds.ac.uk.

ORCID

Owen Cornwell: 0000-0002-7566-9927

Sheena E. Radford: 0000-0002-3079-8039

Alison E. Ashcroft: 0000-0002-1676-123X

\section{Author Contributions}

The manuscript was written through contributions of all authors. All authors have given approval to the final version of the manuscript.

\section{Notes}

The authors declare no competing financial interest.

\section{ACKNOWLEDGMENTS}

The Biotechnology and Biological Sciences Research Council (BBSRC) and AstraZeneca are acknowledged for funding OC (BB/M503459/2). We thank the BBSRC for funding the Compex $\mathrm{KrF}$ excimer laser (Coherent UK Ltd, Ely, UK) (BB/ K000659/1). We thank Dr James Ault, Dr Paul Devine, Dr Matthew Edgeworth, Dr David Lowe, Dr David Brockwell, Prof. Frank Sobott, and members of the Ashcroft and Radford Groups for helpful discussions.

\section{REFERENCES}

(1) Yang, D.; Kroe-Barrett, R.; Singh, S.; Roberts, C. J.; Laue, T. M. $m A$ s 2017, 9 (8), 1231-1252.

(2) Bowen, A.; Casadevall, A. Trends Immunol. 2016, 37 (11), $721-$ 723.

(3) Janda, A.; Bowen, A.; Greenspan, N. S.; Casadevall, A. Front. Microbiol. 2016, 7, 22.

(4) Kiyoshi, M.; Caaveiro, J. M. M.; Kawai, T.; Tashiro, S.; Ide, T.; Asaoka, Y.; Hatayama, K.; Tsumoto, K. Nat. Commun. 2015, 6, 6866.

(5) Wright, J. K.; Engel, J. FEBS Lett. 1978, 90 (1), 79-83.

(6) Wright, J. K.; Engel, J.; Jaton, J. C. Eur. J. Immunol. 1978, 8 (5), 309-314.

(7) Ponomarenko, N.; Chatziefthimiou, S. D.; Kurkova, I.; Mokrushina, Y.; Mokrushina, Y.; Stepanova, A.; Smirnov, I.; Avakyan, M.; Bobik, T.; Mamedov, A. Acta Crystallogr., Sect. D: Biol. Crystallogr. 2014, 70 (3), 708-719.

(8) Pritsch, O.; Hudry-Clergeon, G.; Buckle, M.; Pétillot, Y.; Bouvet, J. P.; Gagnon, J.; Dighiero, G. J. Clin. Invest. 1996, 98 (10), 22352243.

(9) Pritsch, O.; Magnac, C.; Dumas, G.; Bouvet, J.-P.; Alzari, P.; Dighiero, G. Eur. J. Immunol. 2000, 30 (12), 3387-3395.

(10) Lua, W.-H.; Ling, W.-L.; Yeo, J. Y.; Poh, J.-J.; Lane, D. P.; Gan, S. K.-E. Sci. Rep. 2018, 8 (1), 718.

(11) Toughiri, R.; Wu, X.; Ruiz, D.; Huang, F.; Crissman, J. W.; Dickey, M.; Froning, K.; Conner, E. M.; Cujec, T. P.; Demarest, S. J. $m A$ s 2016, 8 (7), 1276-1285.

(12) Stanfield, R. L.; Zemla, A.; Wilson, I. A.; Rupp, B. J. Mol. Biol. 2006, 357 (5), 1566-1574.

(13) Oda, M.; Kozono, H.; Morii, H.; Azuma, T. Int. Immunol. 2003, 15 (3), 417-426.

(14) Sela-Culang, I.; Alon, S.; Ofran, Y. J. Immunol. 2012, 189 (10), 4890-4899.

(15) Koenig, P.; Lee, C. V.; Walters, B. T.; Janakiraman, V.; Stinson, J.; Patapoff, T. W.; Fuh, G. Proc. Natl. Acad. Sci. U. S. A. 2017, 114 (4), E486.

(16) Henderson, R.; Watts, B. E.; Ergin, H. N.; Anasti, K.; Parks, R.; Xia, S.-M.; Trama, A.; Liao, H.-X.; Saunders, K. O.; Bonsignori, M.; 
Wiehe, K.; Haynes, B. F.; Alam, S. M. Nat. Commun. 2019, 10 (1), 654.

(17) Cornwell, O.; Radford, S. E.; Ashcroft, A. E.; Ault, J. R. J. Am. Soc. Mass Spectrom. 2018, 29 (12), 2413-2426.

(18) Zhang, Y.; Wecksler, A. T.; Molina, P.; Deperalta, G.; Gross, M. L. J. Am. Soc. Mass Spectrom. 2017, 28, 1-9.

(19) Calabrese, A. N.; Ault, J. R.; Radford, S. E.; Ashcroft, A. E. Methods 2015, 89, 38-44.

(20) Jones, L. M.; B. Sperry, J.; A. Carroll, J.; Gross, M. L. Anal. Chem. 2011, 83 (20), 7657-7661.

(21) Xie, B.; Sood, A.; Woods, R. J.; Sharp, J. S. Sci. Rep. 2017, 7 (1), 4552.

(22) Xu, G.; Chance, M. R. Chem. Rev. 2007, 107 (8), 3514-3543.

(23) Hambly, D. M.; Gross, M. L. J. Am. Soc. Mass Spectrom. 2005, 16 (12), 2057-2063.

(24) Vahidi, S.; Konermann, L. J. Am. Soc. Mass Spectrom. 2016, 27 (7), 1156-1164.

(25) Niu, B.; Zhang, H.; Giblin, D.; Rempel, D. L.; Gross, M. L. J. Am. Soc. Mass Spectrom. 2015, 26 (5), 843-846.

(26) Rand, K. D.; Zehl, M.; Jørgensen, T. J. D. Acc. Chem. Res. 2014, 47 (10), 3018-3027.

(27) Bai, Y.; Milne John, S.; Mayne, L.; Englander, S. W. Proteins: Struct., Funct., Genet. 1993, 17 (1), 75-86.

(28) Molday, R. S.; Englander, S. W.; Kallen, R. G. Biochemistry 1972, 11 (2), 150-158.

(29) Gau, B. C.; Chen, J.; Gross, M. L. Biochim. Biophys. Acta, Proteins Proteomics 2013, 1834 (6), 1230-1238.

(30) Li, J.; Wei, H.; Krystek, S. R.; Bond, D.; Brender, T. M.; Cohen, D.; Feiner, J.; Hamacher, N.; Harshman, J.; Huang, R. Y. C.; Julien, S. H.; Lin, Z.; Moore, K.; Mueller, L.; Noriega, C.; Sejwal, P.; Sheppard, P.; Stevens, B.; Chen, G.; Tymiak, A. A.; Gross, M. L.; Schneeweis, L. A. Anal. Chem. 2017, 89 (4), 2250-2258.

(31) Yan, Y.; Chen, G.; Wei, H.; Huang, R. Y. C.; Mo, J.; Rempel, D. L.; Tymiak, A. A.; Gross, M. L. J. Am. Soc. Mass Spectrom. 2014, 25 (12), 2084-2092.

(32) Li, K. S.; Chen, G.; Mo, J.; Huang, R. Y. C.; Deyanova, E. G.; Beno, B. R.; O’Neil, S. R.; Tymiak, A. A.; Gross, M. L. Anal. Chem. 2017, 89 (14), 7742-7749.

(33) Shi, L. R.; Liu, T.; Gross, M. L.; Huang, Y. Biochemistry 2019, 58, 1074-1080.

(34) Dobson, C. L.; Devine, P. W. A.; Phillips, J. J.; Higazi, D. R.; Lloyd, C.; Popovic, B.; Arnold, J.; Buchanan, A.; Lewis, A.; Goodman, J.; van der Walle, C. F.; Thornton, P.; Vinall, L.; Lowne, D.; Aagaard, A.; Olsson, L.-L.; Ridderstad Wollberg, A.; Welsh, F.; Karamanos, T. K.; Pashley, C. L.; Iadanza, M. G.; Ranson, N. A.; Ashcroft, A. E.; Kippen, A. D.; Vaughan, T. J.; Radford, S. E.; Lowe, D. C. Sci. Rep. 2016, 6, 38644.

(35) Willis, L. F.; Kumar, A.; Dobson, J.; Bond, N. J.; Lowe, D.; Turner, R.; Radford, S. E.; Kapur, N.; Brockwell, D. J. Biotechnol. Bioeng. 2018, 115 (5), 1216-1225.

(36) Waterhouse, A.; Bertoni, M.; Bienert, S.; Studer, G.; Tauriello, G.; Gumienny, R.; Heer, F. T.; de Beer, T. A P.; Rempfer, C.; Bordoli, L.; Lepore, R.; Schwede, T. Nucleic Acids Res. 2018, 46 (W1), W296W303.

(37) Wilson, I. A., Lang, S., Kurosawa, Y., Ohshima, N., Ota, T., Abadejos, J., Lee, P. S., Nemazee, D. VH1-69 germline antibody predicted from CR6261, 2017, DOI: 10.2210/pdb5WKZ/pdb.

(38) Lobner, E.; Humm, A.-S.; Mlynek, G.; Kubinger, K.; Kitzmüller, M.; Traxlmayr, M. W.; Djinović-Carugo, K.; Obinger, C. mAbs 2017, 9, 1088-1104.

(39) Harris, L. J.; Skaletsky, E.; McPherson, A. J. Mol. Biol. 1998, 275 (5), 861-872.

(40) Brodbelt, J. S. Anal. Chem. 2016, 88 (1), 30-51.

(41) Ballard, K. D.; Gaskell, S. J. J. Am. Soc. Mass Spectrom. 1993, 4 (6), 477-481.

(42) Reis, A.; Fonseca, C.; Maciel, E.; Domingues, P.; Domingues, M. R. M. Anal. Bioanal. Chem. 2011, 399 (8), 2779-2794.

(43) Liu, X. R.; Zhang, M. M.; Zhang, B.; Rempel, D. L.; Gross, M. L. Anal. Chem. 2019, 91 (14), 9238-9245.
(44) Karmakar, T.; Balasubramanian, S. Chem. Phys. Lett. 2014, 613, $5-9$.

(45) Bridgewater, J. D.; Srikanth, R.; Lim, J.; Vachet, R. W. J. Am. Soc. Mass Spectrom. 2007, 18 (3), 553-562.

(46) Wen, N.; Brooker, M. H. J. Phys. Chem. 1993, 97 (33), 86088616.

(47) Perrin, D. D. Dissociation Constants of Organic Acids and Bases; Butterworths: London, 1965.

(48) Maleknia, S. D.; Chance, M. R.; Downard, K. M. Rapid Commun. Mass Spectrom. 1999, 13 (23), 2352-2358.

(49) Boys, B. L.; Kuprowski, M. C.; Noël, J. J.; Konermann, L. Anal. Chem. 2009, 81 (10), 4027-4034.

(50) Brumshtein, B.; Esswein, S. R.; Landau, M.; Ryan, C. M.; Whitelegge, J. P.; Phillips, M. L.; Cascio, D.; Sawaya, M. R.; Eisenberg, D. S. J. Biol. Chem. 2014, 289 (40), 27513-27525.

(51) Rennella, E.; Morgan, G. J.; Kelly, J. W.; Kay, L. E. Proc. Natl. Acad. Sci. U. S. A. 2019, 116 (3), 854.

(52) Petritis, K.; Kangas, L. J.; Yan, B.; Monroe, M. E.; Strittmatter, E. F.; Qian, W.-J.; Adkins, J. N.; Moore, R. J.; Xu, Y.; Lipton, M. S.; Camp, D. G.; Smith, R. D. Anal. Chem. 2006, 78 (14), 5026-5039.

(53) Du, M.; Wu, W.; Ercal, N.; Ma, Y. J. Chromatogr. B: Anal. Technol. Biomed. Life Sci. 2004, 803 (2), 321-329.

(54) van der Vliet, A.; O'Neill Charles, A.; Halliwell, B.; Cross Carroll, E.; Kaur, H. FEBS Lett. 1994, 339 (1-2), 89-92.

(55) Chen, J.; Rempel, D. L.; Gau, B. C.; Gross, M. L. J. Am. Chem. Soc. 2012, 134 (45), 18724-18731.

(56) Johnson, D. T.; Di Stefano, L. H.; Jones, L. M. J. Biol. Chem. 2019, 294 (32), 11969-11979. 